\title{
The three phase rectifier with harmonic injection current
}

\begin{abstract}
This paper demonstrates new circuit and concepts for three phase rectifier using harmonic current injection method. The proposed circuit is using active harmonic current injection method with a capacitor bank which is simple and low cost compared to conventional circuit that using a harmonic current injection method with star-delta transformer. This proposed circuit will reduce the total harmonic distortion which is drawn from input current supply. This will produce the waveform for the input current near to sinusoidal wave. A prototype of the proposed circuit had been developed. The comparison of simulation result and measurement result of the prototype shows good agreement between them. In addition, the THD versus output power of the proposed circuit also had been evaluated and compared with other the conventional circuit that using a harmonic current injection method with star-delta transformer. Finally, this paper will give an overview about a new harmonic current injection method that produce a very low THD of input current.
\end{abstract}

Keyword: Three phase bridge rectifier; Total harmonic distortion; Power conversion 